\title{
Multi-rotor tidal stream turbine fence performance and operation
}

\author{
C. R. Vogel ${ }^{\mathrm{a}, *}$, R. H. J. Willden ${ }^{\mathrm{a}}$ \\ ${ }^{a}$ Department of Engineering Science, University of Oxford, OX1 3PJ, United Kingdom
}

\begin{abstract}
An embedded Reynolds-Averaged Navier-Stokes blade element actuator disk model is used to investigate the performance of a closely spaced cross-stream fence of four turbines. The flow characteristics of such fences are found to be dependent on both the local turbine scale flow problem and the array in channel flow scale problem. The mean fence power is found to be less than that predicted for a single turbine with the same local blockage ratio (ratio of turbine swept area to surrounding flow passage area), but greater than that for a single turbine based on the global blockage ratio of the fence (ratio of total fence swept area to the cross-sectional area of the channel). Cross-fence variation in turbine performance is observed as a result of the differing resistance to bypass flow acceleration around the inboard and outboard turbines and depends on the operating condition of the turbines. Reducing turbine thrust, such as by changing the rotational speed of the turbine or by employing a pitch-to-feather power capping mechanism reduces turbine-turbine interactions and turbine performance becomes more uniform across the fence. An approximately $6 \%$ increase in the mean fence power can be achieved if a cross-fence differential blade pitch strategy is employed to maximise the lift to drag ratio along the majority of the blade span of each of the turbine blades.
\end{abstract}

Keywords: Tidal stream turbines, Tidal turbine fence, Power capping, Blockage effect

1 The importance of the blockage ratio, $B_{L}$, the ratio of rotor swept area to the cross-sectional area of the surrounding flow passage, was demonstrated for tidal stream turbines by Garrett and Cummins [2]. Analysing idealised turbines, it was shown that the peak power coefficient increases by a factor of $\left(1-B_{L}\right)^{-2}$ above the Lanchester Betz limit, $C_{P, \max }=16 / 27$, established for an actuator disk in a unconfined flow field. Subsequent work has extended this model to the case of a long fence of actuator disks occupying part of a much larger channel, showing that inter-turbine interference can still result in theoretical performance improvements beyond the Lanchester Betz limit, even when the turbines occupy only a small fraction of the channel cross section $[7,10]$. The theoretical power coefficient limit increases as a consequence of a streamwise static pressure (head) difference developing in the flow passage as a result of momentum extraction by the actuator disk and mass conservation requirements. The static pressure difference that can be supported in the flow passage increases with the blockage ratio, and thus the increase in maximum power coefficient is achieved at higher thrust levels and lower through-disk flow speeds.

Exploiting the theoretically achievable performance improvements due to blockage ratio with real rotors requires that turbines are specifically designed to sustain the required higher levels of thrust predicted in the analytic models. Two methods by which this can be achieved is increasing the rotational speed of the rotor, or increasing blade solidity in order to increase the generated forces. Cavitation inception concerns at high rotational speeds have restricted the former approach, whereas the latter approach requires blade redesign. Schluntz and Willden [9] investigated computationally the effect of the blockage ratio on the rotor design and performance of tidal stream rotors in an infinitely long fence using Reynolds-Averaged Navier-Stokes (RANS) embedded blade element actuator disk simulations. It was found that required rotor solidity increased and blade pitch angle decreased as the rotors were designed for higher levels of blockage.

\footnotetext{
* Corresponding author

Email address: christopher.vogel@eng.ox.ac.uk (C. R. Vogel)
} 
a)

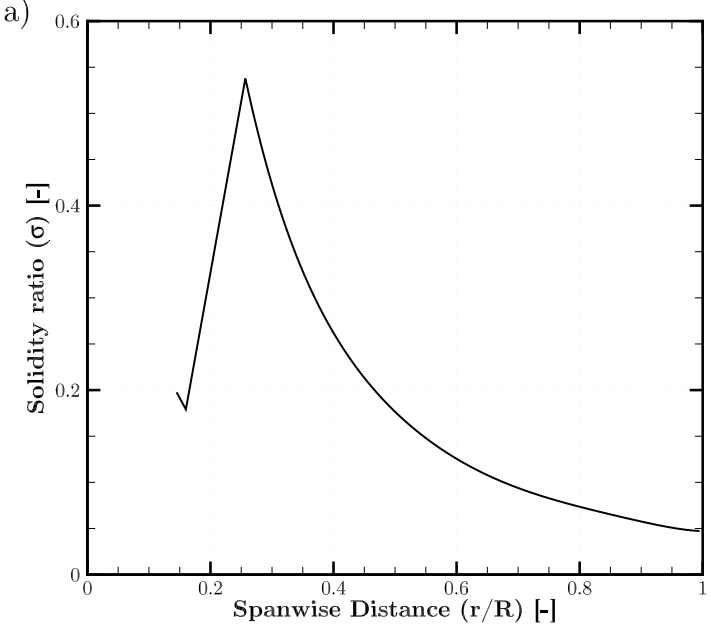

b)

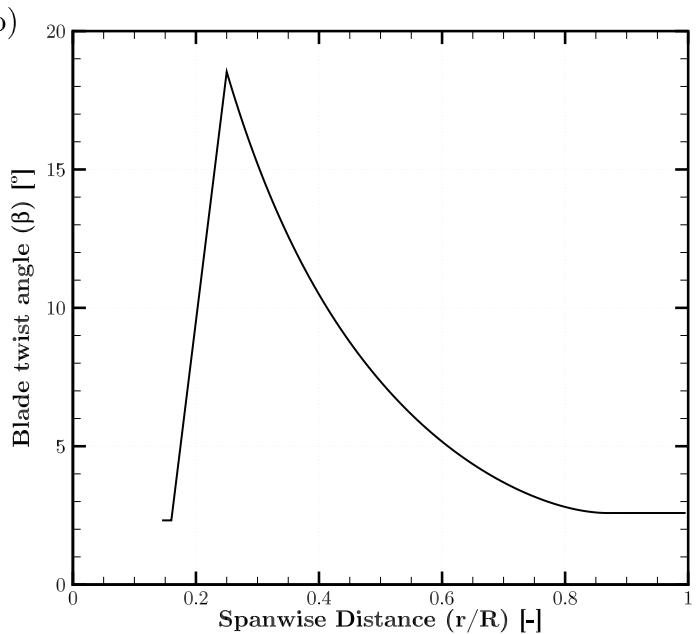

Figure 1: Spanwise blade solidity ratio, $\sigma,(\mathrm{a})$ and blade twist angle, $\beta$, (b) for the $s / d=1$ turbine from [9] and modified by $[11]$.

Cross-stream variations in thrust and power associated with the design of short turbine fences have been highlighted in recent work. Actuator disk experiments by [1] indicate that thrust and power reduce towards the ends of turbine fences, which is detrimental to the overall power of the fence. Similar effects were discussed in an analytic and computational framework by [8], where an analytic model, parameterised by results from numerical simulations, was developed to account for the non-uniform flow speed that is observed across the fence. However, there lacks at present a simple, tractable model to predict cross-stream thrust and power variations across tidal turbine fences and allow design and operation consequences for tidal stream turbine design to be evaluated.

The aim of the present study is to better understand the effect that operating turbines in a short multiturbine fence configuration has on the thrust and power of the individual turbines, as well as the overall turbine fence. An interesting question to be answered is the potential for cross-fence control strategies to improve overall fence performance, such as through cross-fence differential pitching of the turbines. A further question is how such control strategies might change above rated flow speed, when the turbines are operated away from their design conditions. To address these questions, three dimensional (3D) RANS simulations of flow around single and multiple turbines in a range of operating conditions have been conducted.

\section{Rotor selection and computational setup}

The rotor data used herein is taken from [9] for the inter-turbine tip-to-tip spacing ratio $s / d=1$ case, which represents a moderate level of blockage $\left(B_{L}=0.196\right)$. The rotor blades are reasonably thick, using the Ris $\varnothing$-A1-24 aerofoil section along the entire span of the blade. The predicted power and thrust coefficient values in the original work have been shown to correspond well to those derived from high resolution blade resolved and actuator line simulations performed by [11]. Profiles of the blade twist angle, the local angle of the blade chord to the rotor plane, and solidity ratio, the local proportion of rotor circumference occupied by the blades, are shown in Figure 1, where Wimshurst and Willden made modifications to blade geometry near the blade root as required to generate a 3D mesh of the blade. The rotor has three blades, and has a diameter, $d$, of $20 \mathrm{~m}$, and the nacelle has diameter $d_{h u b}=3 \mathrm{~m}$. The nacelle has a hemispherical nose and tail, and is $0.5 d$ in length.

The numerical simulations in this study were performed as steady computations using the commercial CFD solver ANSYS Fluent 15.0, solving 3D incompressible RANS equations with a finite volume method. Turbulence closure was provided by the two-equation $k-\omega$ SST model, which combines the advantages of 


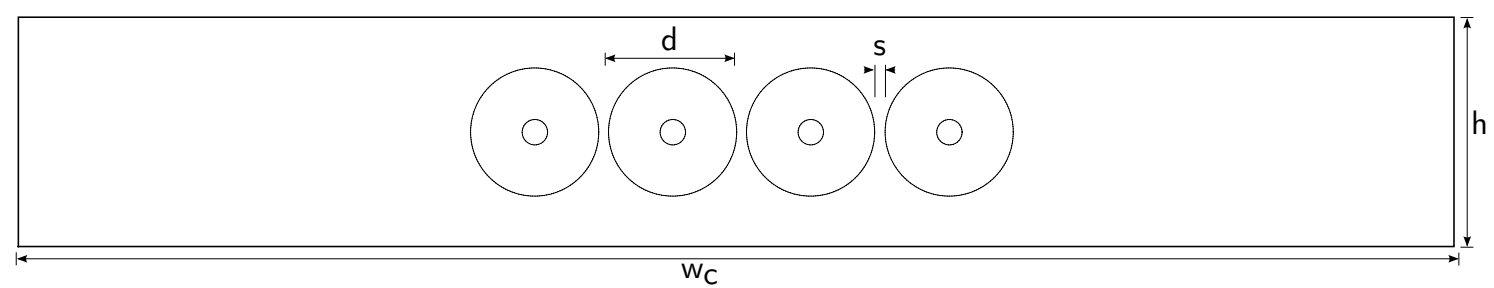

Figure 2: Diagram of the computational domain for the four rotor fence at the rotor plane. $d$ is turbine diameter, $s$ is the inter-turbine spacing, $h$ is the channel depth, and $w_{c}$ is the channel width.

the $k-\omega$ model near no-slip boundaries, such as the nacelle, with the $k-\epsilon$ model in the remainder of the domain [6]. The $k-\omega$ SST model has been used successfully in both marine and wind turbine studies [4].

The tidal turbines were modelled using actuator disks, implemented using the 'porous jump' internal boundary condition in Fluent. A RANS-embedded Blade Element actuator disk (RANS-BE AD) model was specified with user-defined functions following [5], which sampled the flow field at the location of the actuator disk. Flow field data, together with the rotor geometry and aerofoil data, allow the axial and tangential forces to be calculated from blade element theory. The resulting forces are imposed on the flow as a static pressure discontinuity and change in swirl velocity across the disk. The modelling difference between the three-bladed rotor and the actuator disk being simulated (which is an approximation of a rotor with infinitely many blades) was accounted for using the Glauert implementation of the Prandtl tip loss model, see [3]. The tip loss model attempts to reconcile the difference in flow speed that is incident on the rotor blades with the azimuthally-average flow speed through the actuator disk, and is applied to calculations using the axial flow speed sampled from the Fluent simulation.

A rectangular computational domain was used for the simulations. For the single turbine simulations, the actuator disk modelling the tidal turbine was positioned at the centre of the channel cross-section ( $y z$ plane) at $x=0$. The domain extended $10 d$ upstream and $20 d$ downstream of the disk in the $x$ direction. Two blockage ratios were simulated; the first corresponding to the fence local blockage ratio $B_{L}=\frac{1}{4} \pi d^{2} /(s+d) h=0.196$, where $h$ is channel depth and $s$ is the tip-to-tip inter-turbine spacing, and the second corresponding to the fence global blockage ratio, $B_{G}=\frac{1}{4} n \pi d^{2} / w_{c} h=0.0507$, where $n$ is the number of turbines and $w_{c}$ is channel width. In both single turbine cases the channel depth was $2 d$, with the channel width being $2 d$ in the former case, and $7.75 d$ in the latter.

Figure 2 illustrates the fence configuration in the multi-rotor simulations. The channel depth was unchanged at $h=2 d$, and the inter-turbine spacing was $s=d$, such that the local blockage ratio $B_{L}=0.196$. The overall width of the channel was $w_{c}=31 d$. In both the single rotor and multi-rotor simulations, slip wall boundary conditions were used on the upper and lower walls of the domain, meaning that the fluid surface was modelled as a rigid lid, and symmetry boundary conditions were used on the lateral walls. A uniform velocity profile of $u_{\infty}=2 \mathrm{~ms}^{-1}$ was applied to the inlet with seawater $\left(\rho=1025 \mathrm{kgm}^{-3}\right)$ prescribed as the fluid, with a dynamic viscosity, $\mu=0.001 \mathrm{~Pa}$.s, giving a Reynolds number based on the disk diameter of $\operatorname{Re}=4.1 \times 10^{7}$. With no shear to sustain it, the turbulence intensity decreased monotonically from c.15\% at the inlet to c. $4 \%$ at the rotor plane. The static pressure was prescribed to be constant gauge pressure at the outflow boundary.

The computational domains for the single rotor and multi-rotor simulations were discretised with a blockstructured hexahedral mesh. A grid sensitivity study was conducted to determine the resolution required to achieve grid-independent solutions for the integrated power and thrust coefficients, as well as the spanwise variation in angle of attack, and the axial and tangential forces. The final resolution of the mesh had a minimum cell dimension of $d / 200$ and a geometric growth rate of $<1.2$ at the disk perimeter in order to resolve the shear layer that develops at the edge of the actuator disk. The single turbine domain contained approximately $2.5 \mathrm{~m}$ cells, and the multi-rotor domain contained approximately $7 \mathrm{~m}$ cells. 

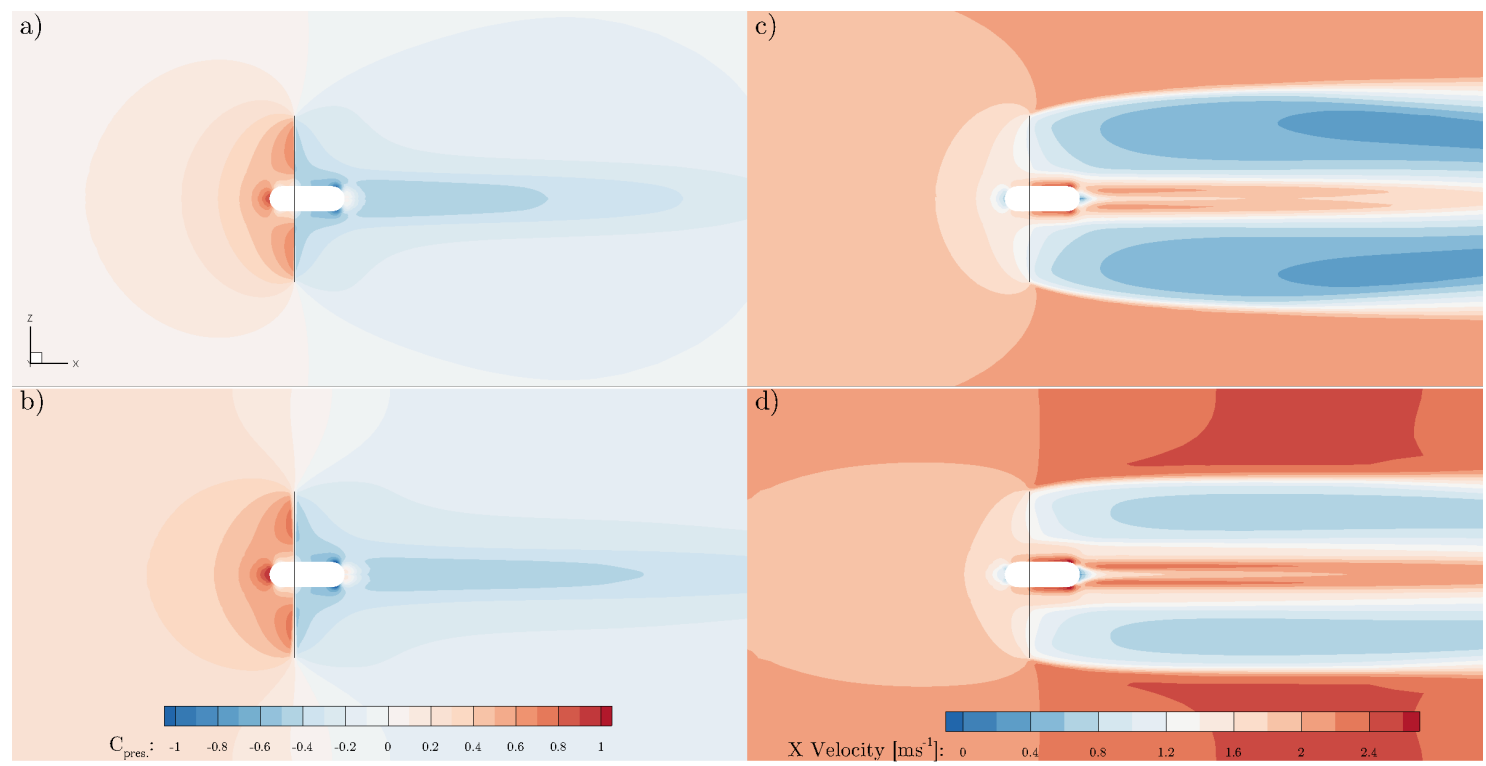

Figure 3: Hub-height slices showing pressure coefficient, $C_{\text {pres }}$ (a \& b) and streamwise velocity $u$ (c \& d) contours for the single rotor in a blockage ratio of $B_{L}=0.0507$ ( $\mathrm{a} \& \mathrm{c}$ ) and $B_{L}=0.196$ (b \& d). Rotor tip speed ratio is $\lambda=5$, and upstream flow speed is $u_{\infty}=2.00 \mathrm{~ms}^{-1}$.

\section{9}

90

\section{Single rotor performance}

Figure 3 compares the spatial variation at hub-height in pressure coefficient, $C_{\text {pres }}=\left(p-p_{\infty}\right) / \frac{1}{2} \rho u_{\infty}^{2}$, and streamwise velocity $u$, where $p$ is the static pressure, $p_{\infty}$ is the upstream static pressure, and $\rho$ is the fluid density, for the two single turbine blockage rotor cases, operating at a tip speed ratio $\lambda=\Omega R / u_{\infty}=5$, where $\Omega$ is the rotational speed of the rotor, $R=d / 2$ is the blade tip radius, and $u_{\infty}$ is the upstream (reference) flow speed. As shown in the figure, the static pressure difference that develops across the rotor depends on the degree of flow confinement in the domain that arises through the proximity of the domain boundaries. The flow bypassing the turbine is accelerated as it passes the flow constriction presented by the rotor and domain boundaries, and consequently static pressure in the bypass reduces. The static pressure in the bypass flow must equalise with the static pressure in the core flow (the flow that passes through the rotor), far upstream and far downstream of the rotor, and consequently a greater static pressure difference develops across the rotor plane at higher blockage ratios. Hence, for a given rotational speed, the device achieves a higher level of thrust in the higher blockage domain than in the lower blockage domain. Rotor torque, and hence power, is also increased in the higher blockage domain as a result of the increased blade forces.

Operating at a single tip speed ratio with increasing blockage ratio does not realise the full performance improvement that is available at higher blockage ratios. Figure 4 shows the effect that tip speed ratio $\lambda$ has on the integrated rotor power and thrust coefficients; $C_{P}=P / \frac{1}{2} \rho u_{\infty}^{3} A_{d}$ and $C_{T}=T / \frac{1}{2} \rho u_{\infty}^{2} A_{d}$ respectively, where $P$ is rotor power, $T$ is rotor thrust, and $A_{d}$ is the rotor swept area. The power and thrust coefficient are uniformly higher for the rotor in the higher blockage $\left(B_{L}=0.196\right)$ configuration than the lower blockage $\left(B_{L}=0.0507\right)$ case. In the higher blockage configuration, the rotor achieves a maximum power coefficient of $C_{P}=0.739$, with a corresponding thrust coefficient of $C_{T}=1.36$ at tip speed ratio $\lambda=6$, as compared to its performance in the lower blockage for which the maximum power coefficient $C_{P}=0.529$, with a thrust coefficient of $C_{T}=1.04$ at a tip speed ratio $\lambda=5$. For a given rotor, the greater resistance to bypass flow acceleration as the blockage ratio increases means that a higher streamwise flow speed may be sustained through the rotor for a given level of thrust. The resulting higher flow speed incident on the turbine blades means that a higher level of power is achieved, as can be determined from Figure 4, where for a thrust coefficient $C_{T}=1.16$, the rotor in the higher blockage domain achieves $C_{P}=0.69$ at $\lambda=5$, as compared to $C_{P}=0.44$ at $\lambda=6.75$ in the lower blockage domain. Increasing turbine thrust further 
a)

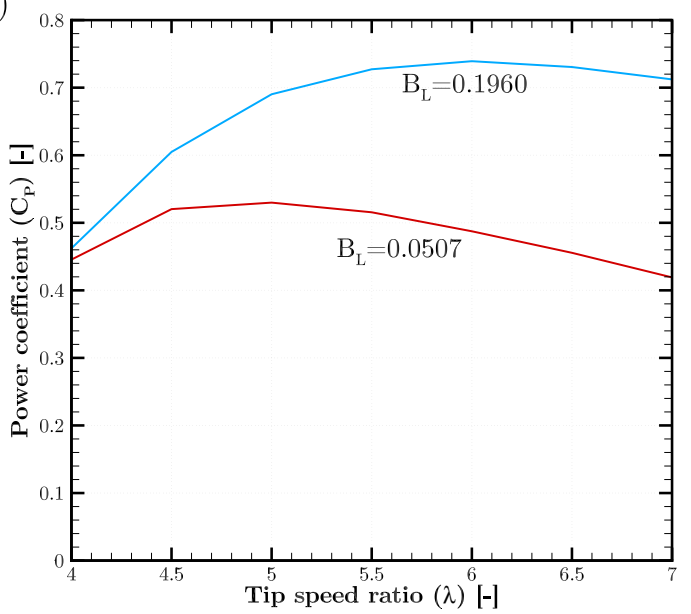

b)

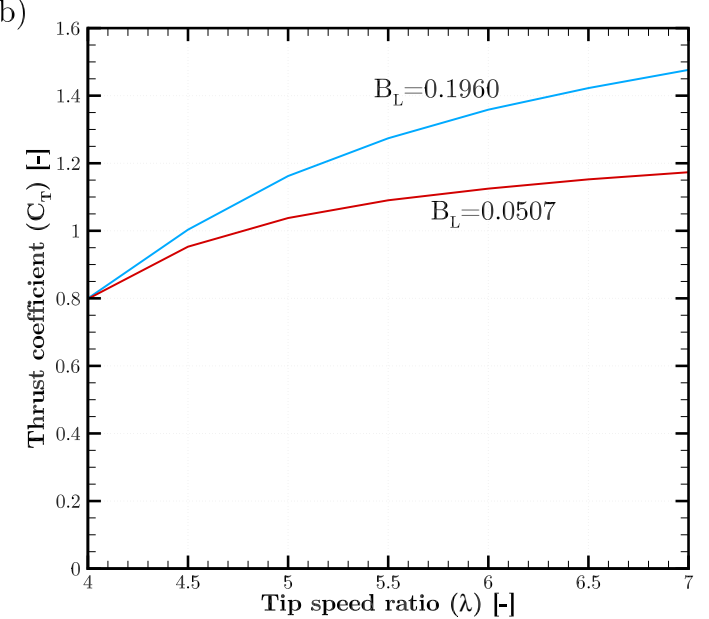

Figure 4: (a) Power coefficient, $C_{P}$, and (b) thrust coefficient, $C_{T}$, variation with tip speed ratio, $\lambda$, for the single rotor in a blockage ratio of $B_{L}=0.196$ (blue) and $B_{L}=0.0507$ (red).

(by increasing the rotational speed of the turbine) results in a reduction of flow through the turbine and a greater acceleration of the bypass flow and consequently a greater streamwise static pressure drop develops in the channel, resulting in an even higher power coefficient being achieved, provided that the thrust is not increased too much. It should be noted that, in general, further increases in power coefficient may be achieved by specifically redesigning the turbine for operation in higher blockage flows and higher tip speed ratios, although cavitation concerns may limit how much the tip speed ratio can be safely increased.

\section{Multi-rotor fence performance}

The four turbine fence, with turbines arrayed in the cross-stream direction as illustrated in Figure 2 with an inter-turbine spacing $s=d$ was simulated in a range of operational conditions. The inter-turbine spacing reflects the local blockage ratio $\left(B_{L}=0.196\right)$ for which the rotor was designed, although the global blockage ratio, which accounts for the fact that the turbine fence occupies only a fraction of the overall channel width, is smaller, $B_{G}=0.0507$. The turbines are numbered from 0-3 left to right across the array, so that turbines 0 and 3 are denoted the 'outboard' turbines, and turbines 1 and 2 are denoted the 'inboard' turbines. The turbines counter-rotate, so that turbines 0 and 2 rotate anti-clockwise, and turbines 1 and 3 rotate clockwise.

Figure 5 presents the effect that tip speed ratio has on the power and thrust coefficient of the inboard and outboard turbines in the multi-rotor array. Although the inter-turbine spacing is the same as in the high blockage single rotor simulations, the maximum inboard and outboard power coefficients $\left(C_{P}=0.535\right.$ and $C_{P}=0.528$ respectively) are significantly reduced from the high blockage single rotor case $\left(C_{P}=0.739\right)$, and much closer to that for the low blockage single rotor case $\left(C_{P}=0.528\right)$. The inboard rotors achieve a slightly higher power coefficient than the outboard rotors, and it can also be seen that the variation in power coefficient with tip speed ratio is reduced as compared to the single rotor cases. Similarly, the thrust coefficient for the inboard and outboard turbines is reduced from that for the high blockage single rotor simulations, but remains higher than that for the low blockage rotor simulations.

Closely spacing the turbines within an array results in the bypass flow around one turbine interacting with the bypass flows around neighbouring turbines, and consequently turbine performance is modified. The interaction of the turbine bypass flows increases the resistance in the bypass flow, and hence the core flow through the turbines is slightly increased, for a given level of thrust, resulting in increased mean power. The increased flow confinement due to neighbouring turbines, as compared to the low blockage single rotor case, 
a)

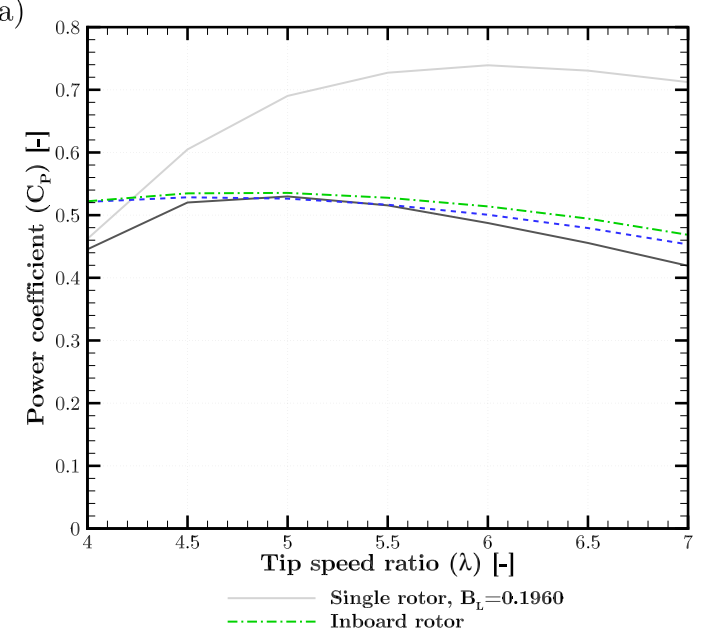

b)

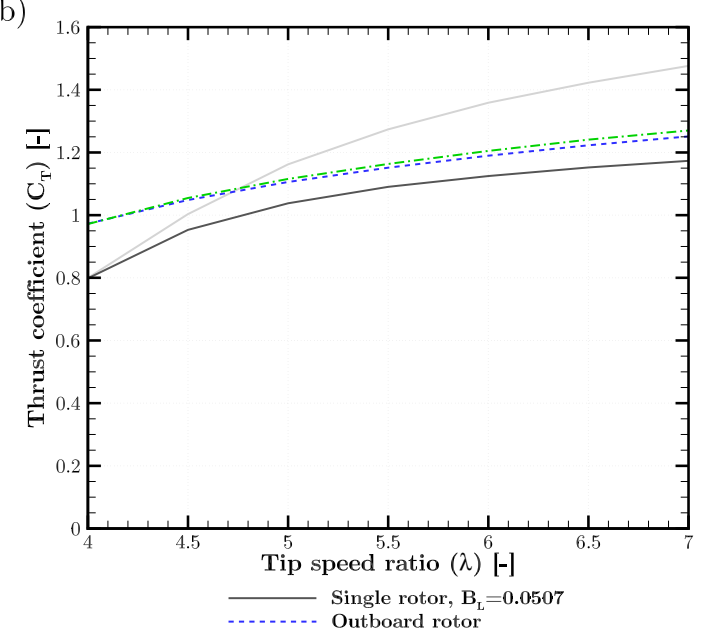

Figure 5: Variation in (a) power coefficient, $C_{P}$, and (b) thrust coefficient, $C_{T}$, with tip speed ratio, $\lambda$, for the inboard (dotdashed) and outboard (dashed) rotors in the multi-rotor fence, with single rotor results shown in grey for reference.

results in a greater pressure drop developing across the array, and hence mean turbine thrust is increased for a given tip speed ratio.

The inboard turbines, which are adjacent to other turbines in both cross-stream directions, experience a greater degree of flow confinement than the outboard turbines, which only experience flow constraint from turbines on one side, with the flow free to expand on the side away from the fence. The bypass flow resistance is hence lower for the outboard turbines, meaning that the core flow is slightly reduced for the outboard turbines as compared to the inboard turbines, and thus the power and thrust coefficients are slightly lower. The magnitude of this difference varies with tip speed ratio, and the thrust and power coefficient difference between inboard and outboard turbines reduces as the tip speed ratio reduces. This phenomenon occurs because turbine thrust reduces with tip speed ratio, and hence the acceleration of the bypass flow is reduced, so that the effect of the bypass flow resistance acting to increase the core flow becomes less significant.

Following [7], two flow scales can be defined; the turbine scale, where flow events occur over spatial distances similar to the turbine diameter, and the larger array scale, where flow phenomena occur over spatial scales similar to the array width. The two scales are linked through the thrust applied to the flow; as the tip speed ratio, and hence thrust, increases, the power coefficient initially increases for both the inboard and outboard turbines, benefiting from inter-turbine interference that allows a higher power coefficient to be achieved than expected given the global blockage ratio in the case of the inboard turbines. As turbine thrust is further increased, the array-scale flow through the fence is reduced due to the overall resistance presented by the fence, resulting in a reduction in mass flow rate through the fence, and hence a reduction in turbine power.

\subsection{Cross-fence differential pitch control}

The change in flow conditions in the multi-rotor fence as compared to the single rotor in the high blockage configuration results in a change in flow angle on the blades of the turbines in the fence. Furthermore, crossstream variations in the streamwise flow speed due to array-scale flow phenomenon mean that the inflow conditions to the inboard and outboard turbines vary. In the high blockage single rotor simulations the angle of attack along the blade span is approximately constant at $\alpha=5^{\circ}$ (as targeted in the design process), which maximises the lift to drag ratio $C_{l} / C_{d}$ for the Ris $\varnothing$-A1-24 aerofoil. Due to the change in flow conditions in the fence configuration, the angle of attack is different from (and greater than) the optimal value for maximising $C_{l} / C_{d}$, and hence rotor power, with the inboard turbines requiring greater correction for the changed flow conditions. 

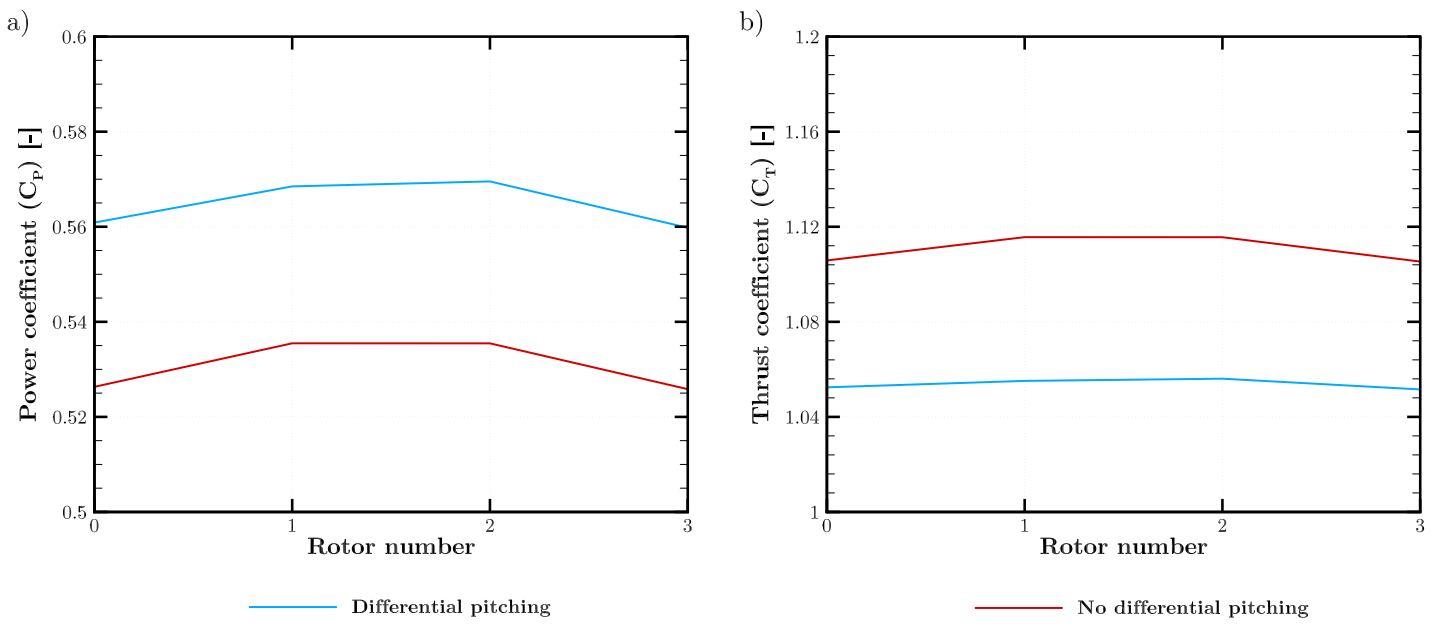

Figure 6: Cross-fence effect of differential blade pitching on (a) power coefficient and (b) thrust coefficient. The change in blade pitch angle for the inboard turbines $\left(1\right.$ and 2) is $\beta_{p}=0.28^{\circ}$ and $\beta_{p}=0.20^{\circ}$ for the outboard turbines. The tip speed ratio is $\lambda=5$.

Figure 6 shows the effect of changing the blade pitch angle by $\beta_{p}=0.20^{\circ}$ for the outboard turbines and $\beta_{p}=0.28^{\circ}$ for the inboard turbines to pitch the blades to feather and thus reduce the angle of attack. The change in blade pitch angle was selected to achieve an angle of attack $\alpha=5^{\circ}$ along the majority of the blade span $0.3 \leq r / R \leq 0.85$. Tip loss effects are significant for radial positions $r / R>0.85$, and hence it is less important to target the optimal angle of attack in this region. Targeting the optimal angle of attack along the majority of the blade span results in an increase in the power coefficient from $C_{P}=0.535$ to $C_{P}=0.569$ for the inboard turbines and from $C_{P}=0.528$ to $C_{P}=0.560$ for the outboard turbines, an approximately $6 \%$ increase. However, cross-fence variation in power coefficient is increased slightly from $1.3 \%$ to $1.6 \%$. The thrust coefficient is reduced when the optimal angle of attack is targeted, from $C_{T}=1.12$ to $C_{T}=1.06$ for the inboard turbines and from $C_{T}=1.10$ to $C_{T}=1.05$ for the outboard turbines, a reduction of approximately $5 \%$. Cross-fence thrust coefficient variation is also reduced. Differential pitching therefore represents a simple way of recovering some of the single rotor performance when deployed in a fence configuration, and through targeting each rotor's maximum lift-to-drag reduces turbine loads which will be of structural benefit to the turbines and may reduce fatigue damage rates.

\subsection{Rated power}

Turbine (and fence) rated power is specified, amongst other factors, based on an economic consideration of the time-varying magnitude of the available tidal resource and the costs (including turbine, generator, support structure) associated with harnessing the resource. Below rated power, the objective is to maximise the power coefficient (i.e., extracted power) and the turbine is operated at the tip speed ratio corresponding to the power coefficient. Above rated flow speed, the power coefficient must necessarily reduce in order to maintain a constant overall level of turbine power, as shown in Figure 7. This may be achieved through a variety of mechanisms, the most common of which for wind turbines is pitch-to-feather control, wherein the blade pitch angle is increased in order to reduce the angle of attack, and hence reduce rotor torque. This also reduces the thrust coefficient and can lead to an overall reduction in rotor thrust, depending on the specific rotor design. Herein we also assume that the generator operates at its optimal rotational speed when rated power is achieved, so that the rotational speed of the rotor is held constant above the rated flow speed, and hence the tip speed ratio reduces. A rated power of $500 \mathrm{~kW}$ per turbine is assumed, to give a rated fence power of $2 \mathrm{MW}$, so that rated flow speed is $u_{r}=1.81 \mathrm{~ms}^{-1}$ for the inboard turbines and $u_{r}=1.82 \mathrm{~ms}^{-1}$ for the outboard turbines, based on the peak $C_{P}$ of the respective turbines in the multi-rotor configuration, see Figure 5. The cut-out flow speed is assumed to be $u_{\infty}=3.50 \mathrm{~ms}^{-1}$. 
a)

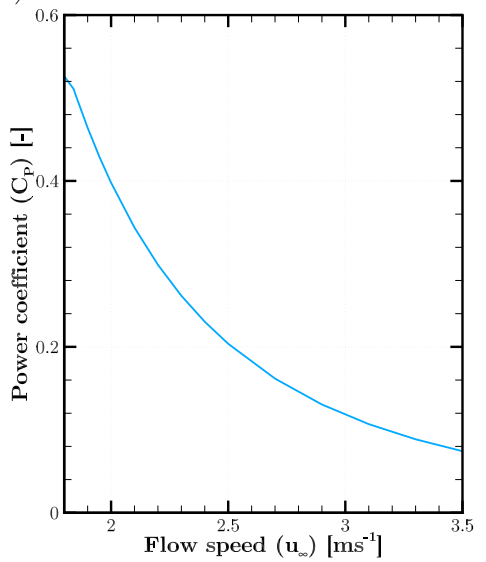

b)

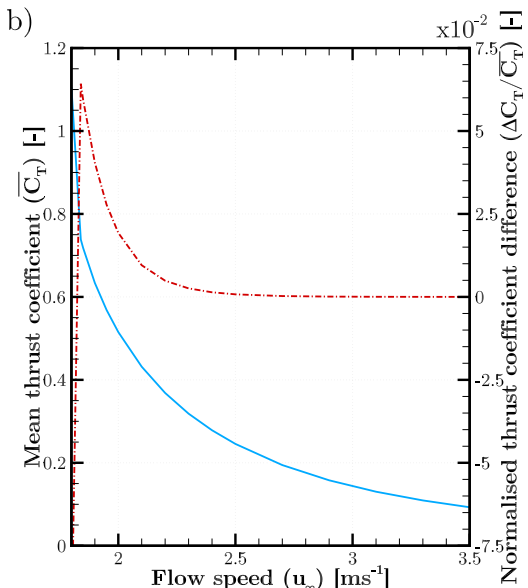

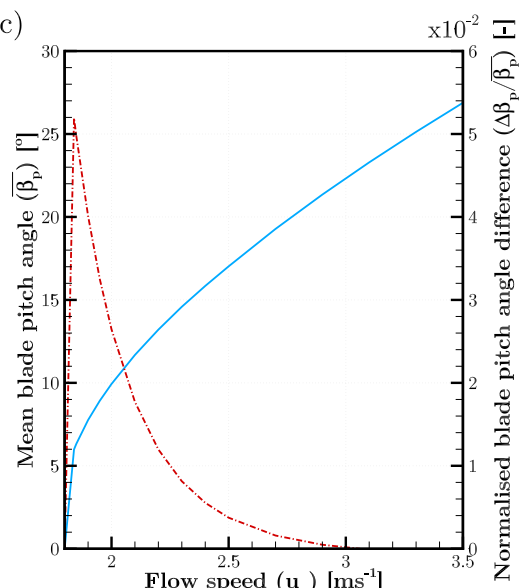

Figure 7: Variation in (a) power coefficient, (b) mean thrust coefficient and normalised cross-fence thrust coefficient difference, and (c) mean blade pitch angle and normalised cross-fence blade pitch angle difference, with flow speed above rated power for pitch-to-feather operation. Rated flow speed of the inboard turbines is $u_{r}=1.81 \mathrm{~ms}^{-1}$ and $u_{r}=1.82 \mathrm{~ms}{ }^{-1}$ for the outboard turbines, and cut-out flow speed is $u_{\infty}=3.50 \mathrm{~ms}^{-1}$.

Figure 7 shows the variation in mean turbine thrust coefficient, $\overline{C_{T}}$, and mean blade pitch angle, $\overline{\beta_{p}}$ with the upstream flow speed, as the blades are pitched-to-feather in order to reduce the power coefficient. The figure also shows the normalised difference in mean thrust coefficient $\Delta C_{T} / \overline{C_{T}}$ and blade pitch angle $\Delta \beta / \overline{\beta_{p}}$ between inboard and outboard turbines as the flow speed increases. The mean turbine thrust coefficient reduces as flow speed increases as the blade pitch angle increases to reduce the angle of attack and hence lift and drag forces. As the inboard turbines reach rated power slightly before the outboard turbines, due to the higher power coefficient of the inboard turbines, there is initially a large difference in thrust coefficient between the outboard and inboard turbines. The outboard turbines operate at below rated power, and hence high thrust, and the inboard turbines begin to increase blade pitch angle and hence reduce thrust. However, the cross-fence thrust variation reduces once both inboard and outboard turbines operate in power capping mode, with the inboard turbines operating at slightly higher thrust than the outboard turbines due to the slightly greater constraint on flow expansion they experience from neighbouring turbines. The cross-fence variation reduces as flow speed increases further and the thrust continues to reduce, and hence the effect of neighbouring turbines on flow expansion is reduced. At the cut-out flow speed, $u_{\infty}=3.50 \mathrm{~ms}^{-1}$, the interference with neighbouring turbines is negligible for reasons discussed further below, so all turbines operate effectively independently and cross-fence thrust coefficient variation is zero.

The mean blade pitch angle increases as the flow speed increases in order to pitch the blades to feather and hence reduce the lift force acting on the blade so as to reduce rotor torque (and hence power). The mean blade pitch angle initially increases rapidly as flow speed is increased, as the lift coefficient, and hence torque-producing lift force (drag is small compared to lift close to the angle of attack which maximises $C_{l} / C_{d}$ ) is large when the angle of attack is close to $\alpha=5^{\circ}$. The rate of change of torque with respect to the angle of attack reduces as the angle of attack reduces, and hence the blade pitch angle changes at a slower rate as flow speed increases further. The normalised cross-fence blade pitch angle variation, $\Delta \beta_{p} / \overline{\beta_{p}}$, reduces as the flow speed increases, as the flow approaching the turbines, and hence rotor power, becomes more homogeneous as thrust reduces.

Figure 8 shows hub-height contours of the streamwise velocity normalised by the reference flow speed for three cases: below rated flow speed, $u_{\infty}=1.80 \mathrm{~ms}^{-1}$ and $\lambda=5.00$, slightly above rated flow speed, $u_{\infty}=2.00 \mathrm{~ms}^{-1}$ and $\lambda=4.55$, and just before the cut-out flow speed is reached, $u_{\infty}=3.50 \mathrm{~ms}^{-1}$ and $\lambda=2.60$. The flow contours reveal that there is significant flow diversion and interaction between turbines in the fence just below the rated flow speed when the turbines operate to maximise the power removed from the flow. At the arrayscale, the streamtube encompassing the flow that passes through the fence expands significantly due to the relatively high level of resistance the fence presents to the flow. The flow immediately upstream of the fence is 
a)

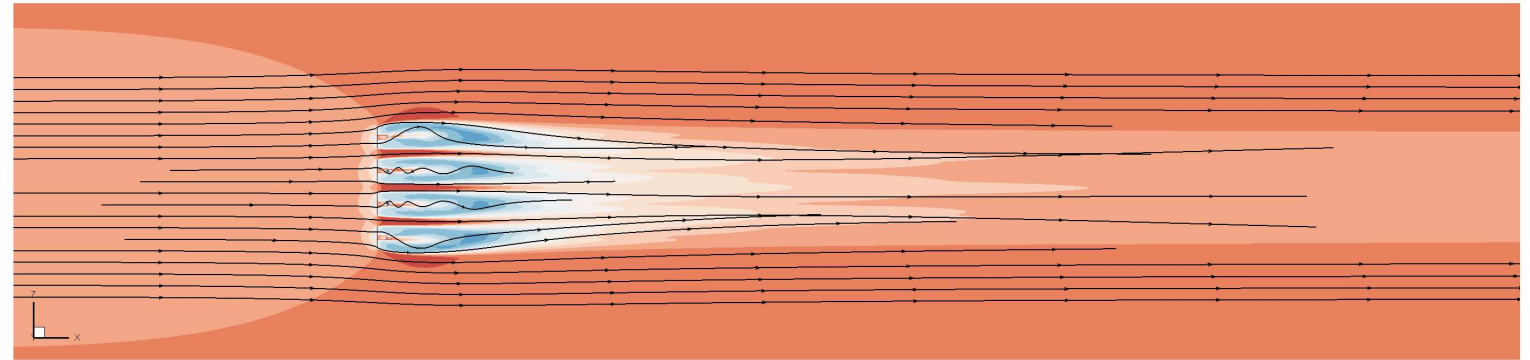

b)

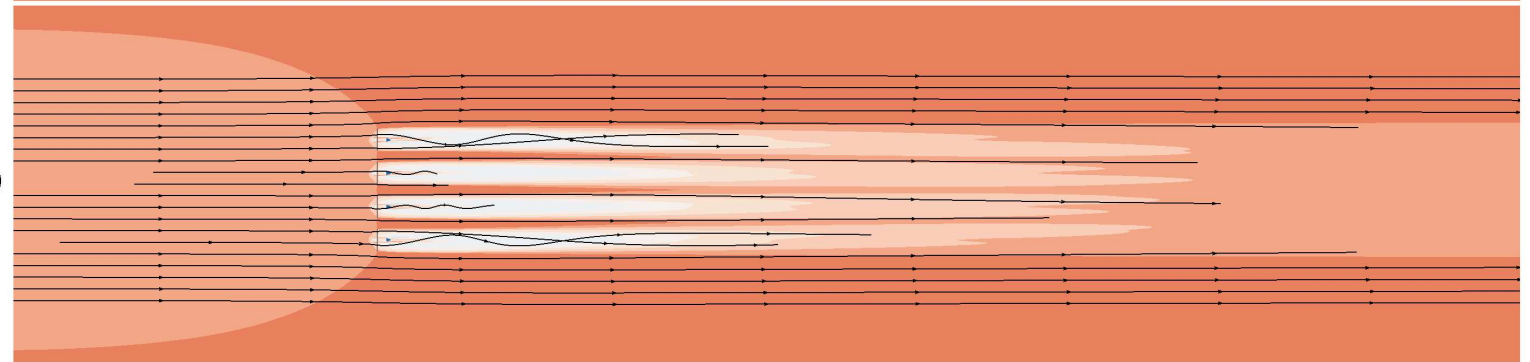

c)

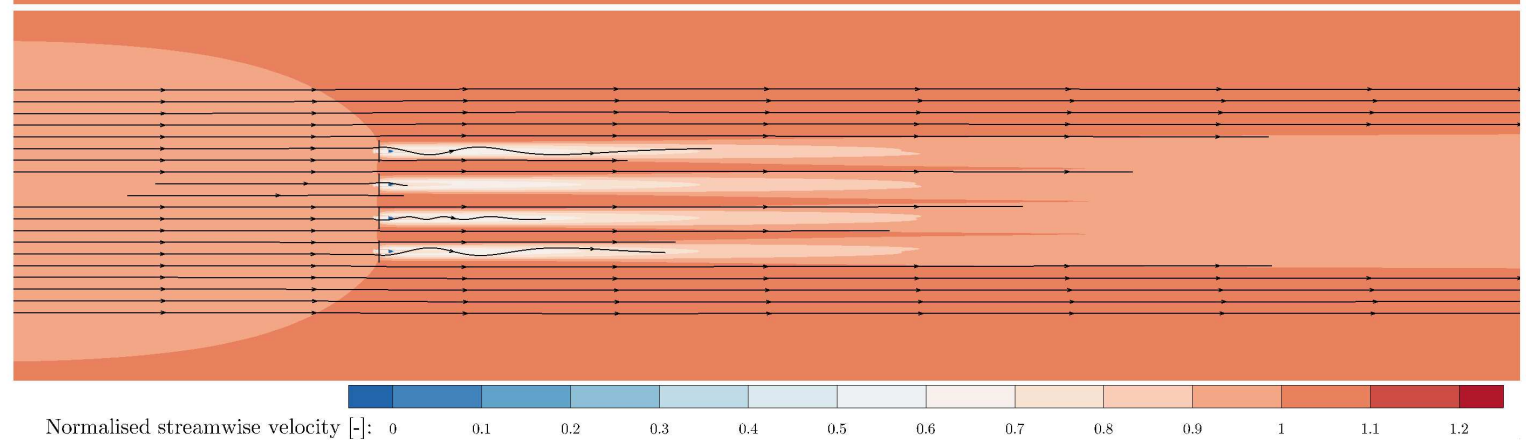

Figure 8: Hub-height streamwise velocity normalised by the reference flow speed for three cases: a) $u_{\infty}=1.80 \mathrm{~ms}{ }^{-1}$, b) $u_{\infty}=2.00 \mathrm{~ms}^{-1}$, and c) $u_{\infty}=3.50 \mathrm{~ms}^{-1}$. Streamtraces have been added to illustrate flow expansion in each case.

decelerated, and the flow in the array-scale bypass accelerated as it passes the fence location, and there is an extended region of flow remixing behind the turbines. At the turbine scale, there is a substantial reduction in flow speed immediately behind the turbines and acceleration of the flow in the inter-turbine bypass regions, and it can be seen that there is asymmetry where the bypass flow expands more freely around the outside of the outboard turbines than around the inboard turbines. Above rated flow speed at $u_{\infty}=2.00 \mathrm{~ms}^{-1}$, the impact that the turbines have on the flow, and hence flow diversion around the array, is significantly reduced. The reduction in turbine thrust above rated flow speed means that the difference in bypass flow resistance between the inboard and outboard turbines is reduced, and consequently cross-fence variations in flow speed, and hence power, are also reduced. As the cut-out flow speed is approached, the turbine resistance imposed on the flow is very small, and hence array-scale flow diversion is negligible. At low thrust levels little flow is diverted around the turbines and hence the effect of the turbines on the resistance to bypass flow acceleration is negligible, meaning that turbine performance characteristics are independent of position of the turbine within the fence. Consequently turbine performance is a function not only of relative turbine placement, but also turbine thrust, and hence turbine operating strategy.

\section{Conclusions}

Garrett and Cummins [2] demonstrated the importance of the blockage ratio in determining the theoretical limit of the power of a tidal turbine in a constrained flow passage, and showed that the power coefficient could 
be increased by a factor of $\left(1-B_{L}\right)^{-2}$ above the Lanchester-Betz limit, if sufficient resistance was applied to the flow. Although a tidal turbine will not achieve the full performance improvement implied by the idealised analysis of Garrett and Cummins due to a variety of hydrodynamic limitations, the physical mechanism in which blockage leads to a greater streamwise static pressure difference remains unchanged. In the case of a single device in a blocked flow passage, the implications of this result are reasonably clear; a higher power coefficient than the Lanchester-Betz limit can be achieved if the device is designed and operated in such a way as to exploit the additional streamwise static pressure gradient that develops in a blocked channel in order to increase rotor torque, and thus power. Single rotor simulations in two blockage ratios, $B_{L}=0.196$ and $B_{L}=0.0507$, were conducted, which exhibited maximum power coefficients of $C_{P}=0.739$ and $C_{P}=0.529$ respectively.

It is not straightforward to define an appropriate blockage ratio to describe the performance increment due to flow confinement effects in the case of a fence of turbines, arrayed normally to the flow direction, that occupies a fraction of a wider tidal channel. A four turbine array was simulated in which the inter-turbine spacing resulted in a local blockage ratio equal to that of the higher blockage single turbine simulation, $B_{L}=0.196$, but the fence of turbines occupied only a small fraction of the larger channel cross-section, so that $B_{G}=0.0507$. Turbine-turbine interference effects meant that the turbine thrust and power coefficients were greater than that predicted for the single turbine, low blockage case, but less than that predicted for the single turbine, high blockage case. This arises as a consequence of the multi-scale flow phenomenon in the channel; the overall resistance the turbine fence presents to the flow reduces the mass flow through the fence and hence that presented to the turbines, limiting turbine power. However, the mutual interference between adjacent turbines acts to reduce the acceleration of flow within the shared bypass flow region, increasing the flow through the turbines, thereby increasing turbine power. The overall effect is a combination of these two opposing dynamics.

The change in incident flow characteristics experienced by the turbine between single turbine and multiturbine fence operation means that it may be beneficial to specify different operating strategies when turbines are deployed in fence configurations. A performance decrement was observed when the turbine, designed to operate in a relatively high local blockage $B_{L}=0.196$, was deployed in a four turbine array configuration. The flow experienced by the turbines was altered due to the additional array-scale flow phenomenon resulting in some flow diverting around the array, reducing the flow speed through the turbines, and consequently the maximum power coefficient was reduced from $C_{P}=0.739$ to $C_{P}=0.528$ for the outboard turbines and to $C_{P}=0.535$ for the inboard turbines. A subsequent $6 \%$ approximate improvement in power coefficient to $C_{P}=0.56$ could be achieved in the fence configuration when the blade pitch angle was adjusted by $\beta_{p}=0.20^{\circ}$ and $\beta_{p}=0.28^{\circ}$ for the outboard and inboard turbines respectively in order to re-target the angle of attack to maximise $C_{l} / C_{d}$ along the majority of the blade span (which was the design condition for the rotors in single turbine operation).

It has been shown that the degree of inter-turbine interference, and therefore the performance improvement available to turbines as a result of blockage effects, depends not only on relative turbine spacing, but also the level of thrust applied by the turbines. Flow confinement, due to adjacent turbines as well as the presence of the sea surface and sea bed, allows an increased streamwise static pressure gradient to develop as mass continuity and energy considerations within the flow passage requires a reduction in static pressure in the bypass flow, and hence a reduction in static pressure far downstream of the rotor. For a given bypass flow speed, and therefore a given static pressure change far downstream of the rotor, a higher turbine thrust can be supported in a more confined flow passage, and thus a higher level of turbine power is achieved. Conversely, the bypass flow speed changes more significantly with turbine thrust for lower blockage ratios. Therefore, as turbine thrust is reduced, the change in the bypass flow speed and therefore interaction with adjacent turbines is reduced. A change in turbine thrust can be achieved through a change in rotational speed as well as a change in blade forces through modification of the blade pitch angle, such as in a pitchto-feather rated power control strategy. Thus cross-fence load and power variations in a short tidal fence depend not only on relative turbine placement, but also turbine operating strategies. For turbines operating with conventional pitch-to-feather power capping strategies it is likely that turbine-turbine interference will be maximum around the rated flow speed. 
[1] Cooke, S. C., Willden, R. H. J., Byrne, B., Stallard, T. and Olczak, A. [2015], Experimental investigation of tidal turbine partial array theory using porous discs, in 'Eleventh European Wave and Tidal Energy Conference', Nantes, France.

[2] Garrett, C. and Cummins, P. [2007], 'The efficiency of a turbine in a tidal channel', Journal of Fluid Mechanics 588, 243-251.

[3] Glauert, H. [1935], Aerodynamic theory, in 'Airplane propellers', Julius Springer: Berlin, pp. 169-360.

[4] Mahu, R. and Popescu, F. [2011], 'NREL Phase VI rotor modelling and simulation using ANSYS Fluent 12.1', Mathematical Modelling in Civil Engineering 1/2, 185-194.

[5] McIntosh, S. C., Fleming, C. F. and Willden, R. H. J. [2011], Embedded RANS-BEM tidal turbine design, in 'Proceedings of the Ninth European Wave and Tidal Energy Conference', Southampton, United Kingdom.

[6] Menter, F. R. [1994], 'Two-equation eddy-viscosity turbulence models for engineering applications', AIAA Journal 32(8), 1598-1605.

[7] Nishino, T. and Willden, R. H. J. [2012], 'The efficiency of an array of tidal turbines partially blocking a wide channel', Journal of Fluid Mechanics 708, 596-606.

[8] Nishino, T. and Willden, R. H. J. [2013], 'Two-scale dynamics of flow past a partial cross-stream array of tidal turbines', Journal of Fluid Mechanics 730, 220-244.

[9] Schluntz, J. and Willden, R. H. J. [2015], 'The effect of blockage on tidal turbine rotor design and performance', Renewable Energy 81, 432-441.

[10] Vogel, C. R., Willden, R. H. J. and Houlsby, G. T. [2016], 'Effect of free surface deformation on the extractable power of a finite width turbine array', Renewable Energy 88, 317-324.

[11] Wimshurst, A. and Willden, R. H. J. [2016], Computational analysis of blockage designed tidal turbine rotors, in 'Second International Conference on Renewable Energies Offshore', Lisbon, Portugal. 